\title{
Research on Public Spaces Reconstruction of Old Communities under the Concept of Micro- renewal
}

\author{
Duan Xiang $^{1, *}$, Xu Tai-meng ${ }^{1}$,Duan Chen-hao ${ }^{2}$ \\ ${ }^{1}$ School of Engineering and Architecture, Wuhan Ploytechnic University, Wuhan,China \\ ${ }^{2}$ School of Architecture,Southeast University,Nanjing,China
}

\begin{abstract}
Community public space is an important place for daily communication between residents. In the context of the current historical change of "urban double repairs", community renewal has raised new heights, and public space renewal has shifted from the surface of the physical space transformation layer to the protection, inheritance and environmental quality of the community. This article summarizes the concept and principles of micro-renewal, analyzes the related influencing factors of the reconstruction of the old community public space, further proposes the strategy of reconstructing the old community public space and enumerate several typical public space renewal schemes in old communities under the micro-renewal concept.
\end{abstract}

\section{Introduction}

Public space narrowly refers to the outdoor place where people conduct social activities, and broadly refers to the ideology of social relations that combines people, environment, and society ${ }^{[1] . S i m p l e ~ m a t e r i a l ~ t r a n s f o r m a t i o n ~ m a k e s ~ t h e ~ s h a p e ~ o f ~}$ community public space the same, lacks features, and public space renewal needs to change its mind.Old communities generally take a long time, the overall space structure is basically complete, the space density is almost saturated, and public spaces need to meet the dual needs of people 's material and spiritual. The "big move" renewal model is obviously not suitable for old communities. The concept of micro-renewal is more in line with the development needs of communities and people, and is the theoretical basis of this study. The reconstruction of public space in old communities is a reconstruction of the relationship between people, society and space, which needs to pay attention to the community's ecological development and humanistic feelings. The purpose of this study is to reconstruct the public space of the old community according to the concept of micro-renewal to promote the sustainable development of the community.

\section{The concept and principle of micro- renewal}

\subsection{The concept of micro-renewal}

"Micro-renewal" is a strategy adopted by the West to abandon large-scale urban renewal and implement urban renewal through small-scale renewal. Micro-renewal is a small-scale, gradual, low-impact update method. The update behavior is closer to the user's space, and it is more able to find problems in community development and reflect residents' demands.Micro-renewal is a long-lasting update process. The project is constantly adjusted and updated according to the needs of the times and life to meet the requirements of use.

\subsection{The principle of micro-renewal}

In micro-renewal, the scale and cost of the project are reduced, which lower the risk of the project. Micro-renewal focus on areas that are more connotative, close to the crowd, and more refined in all aspects[2].The concept of micro-renewal emphasizes that "people-oriented" is always implemented during the renewal process, pays attention to the living needs of residents, and encourages the public to participate in the renewal activities to renew and reconstruct the harmonious relationship between people, environment and society. Micro-renewal respects ecology, inherits historical culture, develops local characteristics, and pays attention to the project's flexible transformation and self-adjustment during and after the renewal process.

\section{Influencing factors of the reconstruction of public spaces in old communities}

\subsection{User's needs}

The main subjects closely related to the community are government, non-governmental organizations and community residents. Based on multiple subjects, listening to the voices of all parties and promoting social equity, participation of multiple subjects is an important feature of micro-renewal and reconstruction. Relying only on government forces can often only form temporary actions, and the update results only stay on the surface. Government power should shift from control to guidance, improve government service awareness and level, and listen to the opinions of residents. Only by fully and truly understanding the needs of community residents can we truly understand the problems in community development and grasp the main contradictions in community development. 


\subsection{The current situation of public spaces in old communities}

Due to the erosion of wind and rain and the disruption of urban development, the explosion of population and the change of people's lifestyles, most old communities have gradually become

Table1. The current situation of public spaces in old communities

\begin{tabular}{|c|l|}
\hline Type & \multicolumn{1}{|c|}{ Explanation } \\
\hline Community lacks public space & $\begin{array}{l}\text { The construction standards of the old community were mainly economic and practical. The } \\
\text { internal density of the community was large, and there was no public space available for } \\
\text { residents 'activities. Most of them were linear spaces for streets and lanes with } \\
\text { transportation functions. }\end{array}$ \\
\hline Low utilization of public space & $\begin{array}{l}\text { The scale of public spaces is relatively small, and most of the public spaces in the } \\
\text { community are used by community residents to dry clothes and pile up waste products. }\end{array}$ \\
\hline Deteriorating public space \\
environment & $\begin{array}{l}\text { The mobile operation of merchant stalls in the old community not only occupies public } \\
\text { space, but the fumes and garbage generated by barbecue stalls and fruit stalls pollute the } \\
\text { environment. }\end{array}$ \\
\hline aglic facilities are imperfect or & $\begin{array}{l}\text { The construction standards of supporting facilities in old communities often cannot meet } \\
\text { the living and living needs of modern people. The quality of road surfaces in the community } \\
\text { varies, the local roads are damaged, subsidence, and water accumulation. Lighting, } \\
\text { maintenance railings, information and other facilities are seriously aging and damaged. }\end{array}$ \\
\hline Low greening rate and poor quality & $\begin{array}{l}\text { Most old communities neglected the construction of greenery and its role in the } \\
\text { construction. In addition to the lack of public space, part of the greenery space was } \\
\text { compressed, making the greening of old communities very poor. Some community } \\
\text { residents independently build flower garden spaces, but the overall landscape quality is } \\
\text { poor }\end{array}$ \\
\hline
\end{tabular}

\subsection{The form and function of public space}

The interface height and interface rhythm of the space form the space form. The height of the space interface is the height of the building surrounding the space. The interface rhythm refers to different forms of building interfaces. The high degree of interface control and rich interface rhythm can make the space full of vitality. Space function is its own vitality and usability. The functions undertaken by community functions are actually the nature and changing patterns of people 's activities. ${ }^{[3]}$.Jane Jacobs clearly put forward the concept of "functional blending" from the perspective of "urban diversity": In a successful and beloved urban area, there are people's activities at different times of the day. In the reconstruction and update of public spaces in old communities, the repair and setting of mixed functions are essential. Repairing the basic functions of the community can make the old community adapt to different activities and provide multilevel comprehensive service functions for local residents and tourists.

\subsection{Inheritance and continuation of public space culture}

Old community buildings are declining, so the buildings bearing the historical and cultural marks may be rudely weak in the city, swallowed by dense large-scale urban development, plus overuse and lack of protection consciousness, the overall appearance of the community is broken. The following table lists the current status of public spaces in old communities (Table1) 
unreasonable roads, improve the accessibility of street spaces, and connect spatial nodes to form a complete spatial sequence, which not only increases the interest of public spaces in old communities, but also provides more communication opportunities and interaction time for residents to go out.

\subsection{Reconstructing the form of public space}

\subsubsection{Maintaining continuity of the interface}

The continuous space interface makes the people coming and going to stay, most people like to stay on the edge of the space and observe the activities in the space. The space formed by the enclosed and continuous boundaries can better guide people's activities and make full use of the combined effect of the space. Therefore, when reconstructing the public space of the old community, we should pay attention to the continuity of the space interface, establish an effective and clear transition within the horizontal horizon of people, and form a virtual and real space through various forms such as bumps, retreats, overhangs to attract people.

\subsubsection{Improving space accessibility}

Space accessibility includes sight accessibility and path accessibility. Sight accessibility can effectively guide people's behavior and have a positive impact on spontaneous activities. When people can see the activities in public places, the combined effect will attract more people to participate. The barrier-free sight will make the activities more natural, and the use of open spaces can be improved to a certain extent. Path accessibility is represented by a walking system, a short walking length, an effective and convenient entry path, and a reasonably unobstructed road. Opening up blocked roadways and using more effective spatial connections can make it easier for people to reach interesting spaces

\subsubsection{Moderately transforming the shape of the space}

Different forms of space will bring different psychological influences and hints to people. Intermittent public space means that people will stop, linear public space means that people will have a tendency to move forward in the space, the enclosed space implies that residents can stay here. According to the community development and the needs of the residents, the appropriate transformation of the space form can enhance the subject's sense of use and satisfaction. Public spaces should try to avoid long and straight lines, so that the walking experience becomes an interesting thing. Creating rhythmic changes on the interface of the ground floor space and cooperating with the landscape greening, will enrich people's space experience.

\subsubsection{Humanized spatial scale}

The spatial form in the old community is more complicated and chaotic. Therefore, in the reconstruction of the public space in the old community, humanized spatial scale should be established and maintained."the hidden dimension"mentions, when the distance exceeds $25 \mathrm{~m}$, it is difficult for people to recognize the language and facial expressions of others, and it is difficult to produce communication.Jan Gehl proposed that $100 \mathrm{~m}$ is the pole of the experience distance ${ }^{[5]}$. For a linear space, a width less than $25 \mathrm{~m}$ can help people passing by to perceive each other better. However, a narrower spatial scale will cause discomfort to the two streams of people who meet in opposite directions. This space is only a temporary space and cannot produce activities such as staying and communicating.

\subsection{Reconstruction of public space functions}

In the long-term development process of old communities, many functional spaces have spontaneously formed, such as sports spaces, farmers 'markets, supermarkets, breakfast shops, etc. These spaces conform to the trajectory of the residents 'lives, and should be retained during the reconstruction of public spaces, not only the needs of the indigenous people 's social relations, but also the necessary conditions for the continuation of the indigenous people 's lifestyle. At the same time, integrating the original function configuration and dismantling the privately built space will make the disordered space function orderly. The function of the original public space in the old community is relatively simple. According to the resources of the community and the needs of the residents, the single function can be replaced with small and medium-sized inclusive functions, and the original functional space can be integrated and reconstructed to give the space new and diverse functions and increase the variety of choices for space users.

\subsection{Reconstructing the continuation of public space}

The spatial structure and scale of the old community have formed a unique space texture in the community for a long time. Streets, courtyards and nodes are the public space of the community. The organic extension of the space texture can be carried out in the following ways. We can precisely control the "scale" of public space, protect historical buildings, and give it new connotation and significance. Also, we can select good quality building samples from the existing public spaces, execute a reasonable transformation process, and replace effective functions with them. Under the low-cost and low-risk concept advocated by micro-renewal, the parts of old communities that need to be demolished can make full use of the original building materials to restore vitality in new buildings and establish an organic connection between old and new spaces. 


\section{Renewal plan for some typical public spaces in old communities under the concept of micro-renewal}

\subsection{Community entrance}

\subsubsection{Space characteristics}

The entrance forms of old communities can be divided into street-style entrances and open entrances. The entrance form of the partial overhead of the first and second floors of the building is the entrance to the street, which residents are more likely to stay chat. Open entrances are more fluid than street entrances, and it is easier to shape the brand characteristics of the community. The disorderly parking of non-motorized vehicles, publicity boards, rest facilities cause a mess in the open entrance.

\subsubsection{Renewal plan}

The main point of updating the street-style entrance is to increase the convenience of residents passing here and the improvement of interpersonal communication. For old communities where is other spaces to park non-motorized vehicles, we should move and park non-motorized vehicles to expand travel space; For old communities where there is no other space to park non-motor vehicles, we should divide the parking places of non-motor vehicles and regulate the parking order.

The main points of the reneal of the open entrance are the community image and the separation of people and vehicles. The entrance image should be combined with the historical and cultural characteristics of the community and coordinated with the architectural style of the community; The pedestrian-vehicle intersection should regulate the diversion route; Publicity boards, rest seats, etc. should not be placed near the entrance to avoid affecting the smooth travel.

\subsection{Street space}

\subsubsection{Space characteristics}

Street space can be divided into street space and roadway space. The street is the widest road in the community, with a large spatial scale, which can be used by vehicles. It is generally a two-lane or single-lane road, with one or two sidewalks attached, which is the link between the community and the outside world. The laneway is the secondary road of the community. The scale of the space is small, usually a single lane or a narrow road only for non-motor vehicles and residents.

\subsubsection{Renewal plan}

The main point of the street space update is to improve the safety and comfort of the space. We should strictly divide the pedestrian and vehicle routes, promote the separation of pedestrians and vehicles, and separate pedestrians and vehicles with green belts to ensure the smooth vision of road intersections, and buildings, facilities, greening, etc. ${ }^{[6]}$ Community motor vehicle lanes with a width of less than $10 \mathrm{~m}$ can regulate the order of motor vehicles parked on the street. Community motor vehicle lanes with a width of more than 10 meters can increase the marking line to subdivide the vehicle space and add non-motor vehicle lane. Also, we should add emergency traffic routes to meet firefighting and first aid. The design of this route should avoid interference with residents' daily routes. Generally, the width of the walkway is about $2 \sim 3 \mathrm{~m}$, and the sidewalk with a width of more than $3 \mathrm{~m}$ can be set on the side of the green belt for the leisure space of the pedestrians.

\subsection{Other functional public spaces}

\subsubsection{Amusement park space transformation}

The quality of the amusement park space in the old community is generally poor, mainly due to the relatively single space form, messy plant species, and outdated leisure facilities. Transformation of amusement park space should be designed according to local conditions, and combined with actual plot status and community needs. We should compact layout, improve land utilization. If the plot area is small, the park road should adopt a regular pattern; if the plot area is large, a natural tortuous path can be used. Through the rational addition of different living and leisure facilities to enrich the functions of the amusement park space; Through plant configuration and landscape transformation to improve the greening quality of the space.

\subsubsection{Sports space transformation}

The renovation and reconstruction of the sports space should focus on practicality and safety.

First of all, according to the main types of activities of community residents such as dance, fitness, etc., rationally divide the venues and update the corresponding sports equipment; Secondly, the venue should pay attention to the anti-slip property of the surface layer, and the sports plastic surface layer. The children's field can be laid with colored plastic surface layer to increase the fun of the venue. Finally, the plant landscape should be properly configured to reduce the impact of pollen and insects on the residents.

\subsubsection{Micro space transformation}

The renovation of the micro-space is based on the current situation, revitalizing the stock, and invigorating the vitality as the starting point, oriented by the problem, and detailed design according to local conditions. For example, the micro space next to the residential building should be cleaned of debris first, and the functional quality of the space should be improved through landscape reconstruction combined with leisure facilities. Street 
green space should focus on reducing the interference of dust, car exhaust, and noise to meet the functional needs of residents' rest and communication. Residents of old communities often spontaneously open up flower garden spaces next to their houses. Due to the difference in the size, planting type, and repair materials of each flower garden space, they appear complicated, complicated, and chaotic. In order to reduce the clutter of the flower garden space, flower pots of the same material and repair materials of the unified flower garden can be used to combine scattered flower and pot plants.

\section{Conclusion}

The ideal spatial form, on the one hand, must inherit the local history and culture, on the other hand, it needs to become a place to effectively gather social relations. Effective public space renewal can inject new blood into the community and promote the community's new vitality. Taking renewal and reconstruction of public space as an entry point is an important way to solve the disorderly development of public space, cultural mining, and reshaping of sustainable social relations in the development of old communities, and also provides a valuable model for the development of public spaces in old communities.

\section{Funding}

This work was supported by the Hubei Social Science Foundation(2018q124), Wuhan Committee of Urban and Rural Construction(201739) and Wuhan Social Science Foundation(19005).

\section{References}

1. Gao Y.B. Analysis of Urban Public Space [J]. Shanxi Architecture, 10 5-6. (2013)

2. Zhu W, Chiu R L H. The Planning and Design of Environmentally Sustainable High-Rises[M]// HighRise Living in Asian Cities. Springer Netherlands,553-560. (2011)

3. Shi S.J. "Environmental micro-renewal" historical location protection model exploration [D]. Southeast University (2015)

4. Lu W. Research on the Humanistic Design of the Landscape Reconstruction of the Old City Community under the Sustainable DevelopmentD]. Northeast Normal University (2014)

5. Jan Gehl,Ouyang W. Cities for People [M]. China Construction Industry Press,(2010)

6. Ren Y, Zhang X.K. Research on Road Landscape Design Method [J]. Urban Construction Theory Research, 17 (2013) 